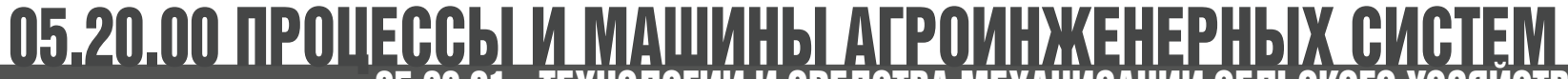

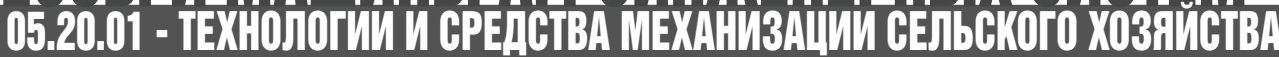

\author{
УДК 631.3
}

DOI 10.18286/1816-4501-2019-1-6-11

\section{ТЕОРЕТИЧЕСКИЕ ИССЛЕДОВАНИЯ ПРОЦЕССА ДОЗИРОВАНИЯ СЫПУЧЕГО МАТЕРИАЛА}

Семашкин Николай Михайлович ${ }^{1}$, кандидаm технических наук, доцент кафедры "Математика и физика»

Исаев Юрий Михайлович ${ }^{1}$, доктор технических наук, профессор, заведующий кафедрой «Математика и физика»

Крючин Николай Павлович², доктор технических наук, профрессор, заведующий кафедрой «Механика и инженерная графика»

Крючин Александр Николаевич ${ }^{2}$ кандидат технических наук, доцент кафедры "Механика и инженерная графика»

${ }^{1}$ ФББОУ ВО УльяновскИй ГАУ

432017, г. Ульяновск, бульвар Новый Венеи, 1; тел.: 8 (84231) 55-95-49, e-mail: emotion.snm@ mail.ru

${ }^{2}$ ФББОУ ВО Самарская ГСХА

446442, Самарская область, п.г.m. Усть-Кинельский, ул. Учебная, 2, e-mail:kryuchin@inbox.ru

Ключевые слова: частица, сыпучий материал, уравнение Навье-Стокса, дозирование, перемещение, пропускная способность.

В статье рассмотрено движение сыпучего материала на основании гидродинамической модели с использованием уравнения Навье-Стокса для вязкой жидкости в векторной форме. Рассмотрен слой перемещаемого материала, толщина которого значительно меньще по сравнению с его длиной и шириной. В теоретической части статьи описываются исследования движения сыпучего материала в дозирующем устройстве, где учитываются площадь и масса материала. А средняя скорость частиц материала ограничивается высотой поднятия штифтов, которая отличается от скорости вращения диска, так как одной из основных характеристик сыпучего материала является коэффичиент внутреннего трения частии материала. При этом для определения пропускной способности дозатора учитывалось выражение для определения средней скорости перемещения частиц материала. Получено уравнение, представляющее собой математическую модель неустановившегося режима движения, и проанализировано с учетом граничных и начальных условий. Найдено отношение скорости перемещения сыпучего материала к линейной скорости транспортера в различных точках по толщине слоя перемещаемого материала в различные моменты времени. В итоге выявлено, что использование итифтов для истечения сыпучего материала из загрузочного окна в дисково-штифтовом дозирующем устройстве позволяет обеспечить значительное увеличение его пропускной способности. Полученные теоретические исследования для определения скорости перемещения сыпучего материала вращающимся диском с подпружсиненными штифтами позволяют получить оптимальную пропускную способность устройства при дозировании частии материала в зависимости от конструктивных параметров этого устройства и физико-механических свойств материала. При максимальной высоте штифтов в средней части зоны загрузки материала пропускная способность устройства не превышала 0,55 кг/с.

\section{Работа выполнена в рамках гранта Президента Российской Федерации для государственной поддержки молодых российских ученых - кандидатов наук МК-6675.2018.8}

\section{Введение}

В сельскохозяйственном производстве большое значение имеет повышение качества дозирования сыпучих материалов. Одними из основных направлений улучшения функционирования дозирующих устройств является теоретическое исследование и моделирование процессов переноса материала. Главным и суще- 
ственным недостатком существующих средств дозирования следует считать высокие энергетические и материальные затраты на выполнение технологического процесса. Это обуславливает необходимость совершенствования средств дозирования сыпучих материалов, оптимизации их параметров и режимов работы, в том числе и на основе анализа математических выражений, описывающих процесс их работы.

Работа рассматриваемого устройства для дозирования сыпучих материалов заключается в следующем. Под действием вращающегося диска штифты движутся по поверхности направляющей шайбы, причем траектория их движения повторяет форму шайбы за счёт подпружиненных опорных концов (рис. 1). Во время движения штифты приподнимаются выше уровня козырька и далее поочерёдно входят в перемещаемый в устройстве материал. Вследствие этого своды, образовавшиеся возле загрузочного окна, разрушаются, и сыпучий материал равномерно распределяется по вращающемуся диску. Затем материал ровным слоем перемещается через выходное окно и скребком сбрасывается в эжекторное устройство [1].

\section{Объекты и методы исследований}

Выполним теоретические исследования движения сыпучего материала в дозирующем устройстве. Учтем, что площадь и масса материала известны. Средняя скорость частиц материала, которая ограничена высотой поднятия штифтов, отлична от скорости вращения диска, так как одной из основных характеристик сыпучего материала является коэффициент внутреннего трения частиц материала. Для определения пропускной способности дозатора необходимо учитывать выражение для определения средней скорости перемещения частиц материала.

Движение сыпучего материала совершается за счет вращения диска (рис. 1). Ось X совпадает с направлением перемещения материала диском, а ось Z направлена вертикально вверх.

Для исследования движения исследуемого сыпучего материала рассмотрим его как вязкую жидкость с насыпной плотностью $\mathrm{\gamma}$, а кинематический коэффициент внутреннего трения частиц материала обозначим через П. Динамику движения материала выразим через уравнение Навье-Стокса для вязкой жидкости в векторной форме [2, 3, 4]:

$$
\vec{F}-\frac{1}{\gamma} \operatorname{grad} P+v \nabla^{2} \vec{v}=\frac{\partial \vec{v}}{\partial t},
$$

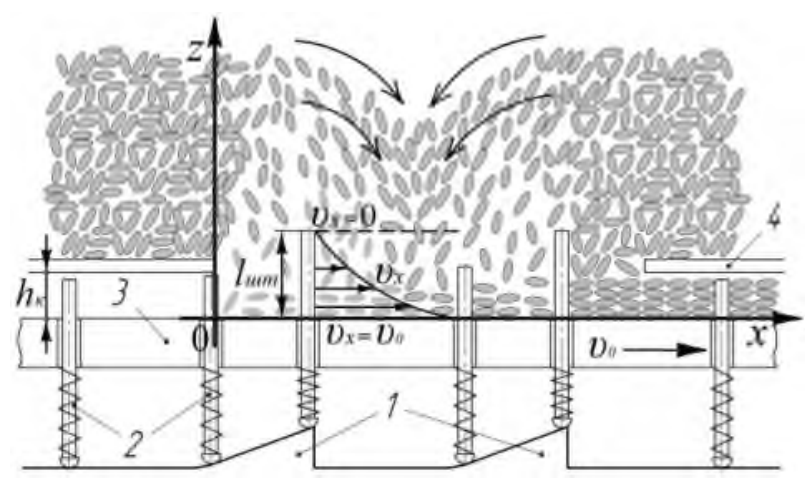

Рис. 1 - Схема распределения скорости материала, перемещаемого по диску:

1 - ступеньки; 2 - подпружиненные штифрты; 3 - вращающийся диск; 4-козырёк

где $\bar{F}$ - сила тяжести, действующая на частицу, H; ү - плотность материала, кг/м³; P нормальное давление на частицу, Па; $v=\eta / \mathrm{v}-$ коэффициент вязкости, м² $^{2}$; $\nabla^{2}$ - дифференциальный оператор Лапласа; u - скорость частицы, м/c; t - время перемещения, с.

Дифференциальный оператор Лапласа имеет вид:

$$
\nabla^{2}=\frac{\partial^{2}}{\partial x^{2}}+\frac{\partial^{2}}{\partial y^{2}}+\frac{\partial^{2}}{\partial z^{2}}
$$

где x, y, z - текущие координаты частицы.

Спроецировав силы, действующие на частицу сыпучего материала по осям системы координат, перепишем уравнение в следующем виде:

$$
\left\{\begin{array}{l}
\frac{d v_{x}}{d t}=X+v \cdot \nabla^{2} v_{x}-\frac{1}{\rho} \frac{\partial P}{\partial x} \\
\frac{d v_{y}}{d t}=Y+v \cdot \nabla^{2} v_{y}-\frac{1}{\rho} \frac{\partial P}{\partial y} \\
\frac{d v_{z}}{d t}=Z+v \cdot \nabla^{2} v_{z}-\frac{1}{\rho} \frac{\partial P}{\partial z}
\end{array},\right.
$$

где $v_{x^{\prime}} U_{y^{\prime}} u_{z}$ - проекции скоростей частицы сыпучего материала на соответствующие координатные оси, $\mathrm{M} / \mathrm{c} ; X, Y, Z$ - проекция массовых сил, $\mathrm{H}$.

Поскольку движение материала совершается при вращении диска, то уравнение движения частицы материала рассматривается относительно подвижной системы координат. Тогда силы инерции, действующие на частицу, учитываются как массовые силы тяжести.

Так как ось X направлена по направлению движения, а ось Z перпендикулярна к оси X, то для режимов движения дозирующего устройства уравнения (2) запишем следующим обра- 
30M:

$$
\left\{\begin{array}{l}
\frac{d v_{x}}{d t}=v \frac{d^{2} v_{x}}{d z^{2}} \\
\frac{1}{\rho} \frac{\partial P}{\partial z}=g
\end{array}\right.
$$

Рассмотрим особенности процесса дозирования в рассматриваемом устройстве:

а) относительная скорость перемещения частиц сыпучего материала во время движения зависит от сил внутреннего трения;

б) относительное перемещение материала зависит от параметров движения диска.

Движение потока частиц описывается первым уравнением, входящим в систему (3), если при этом не учитывать силы инерции перенос-

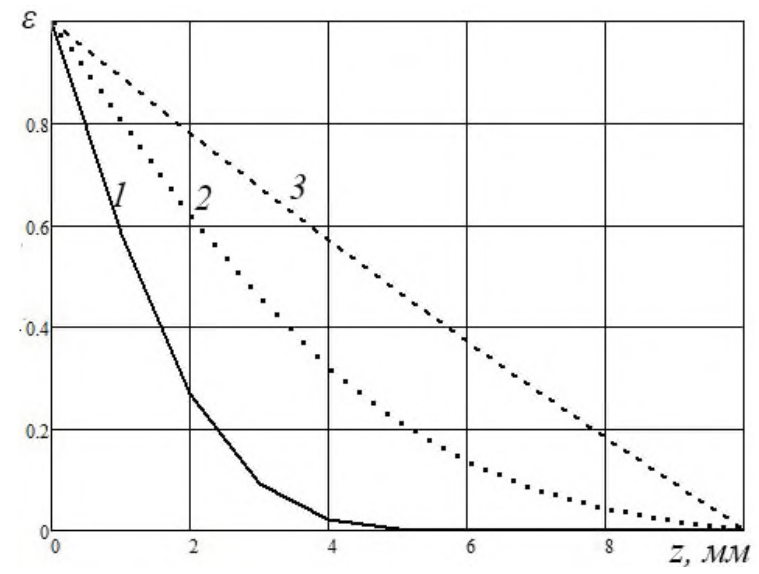

Рис. 2 - Зависимость отношений скоростей $\varepsilon$ от координаты $z$ вдоль оси по толщине перемещаемого слоя в различные моменты времени: $1-t=1 \mathrm{c} ; 2-t=3 c ; 3-t=10 \mathrm{c}$

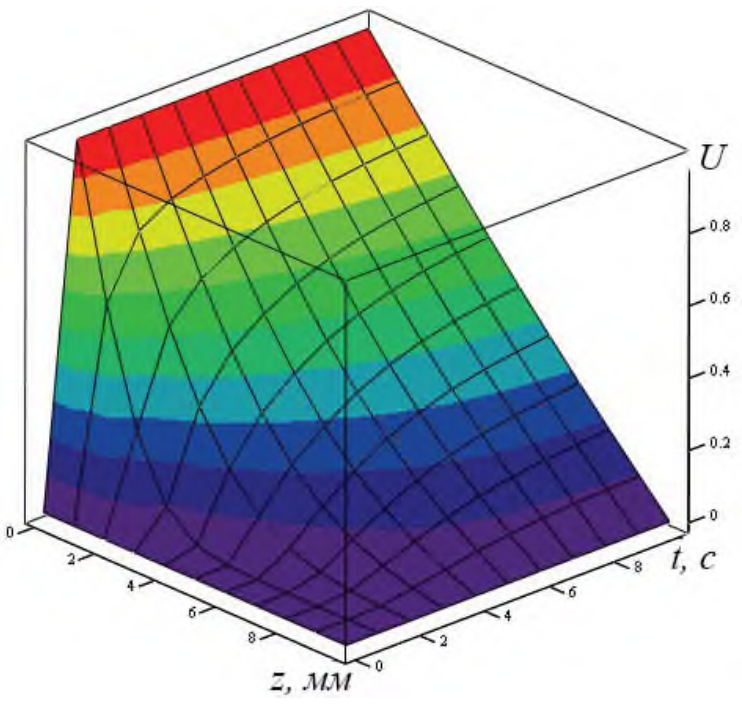

Рис. 3 - Изменение отношения скоростей $\varepsilon$ от координаты $z$ вдоль оси по толщине перемещаемого слоя и времени $t$. ного движения. Преобразуем пространственную задачу в одномерную, тогда дифференциальное уравнение примет вид:

$$
\frac{d v_{x}}{d t}=v \frac{d^{2} v_{x}}{d z^{2}}
$$

Зададим граничные условия движения диска, учитывающие факторы переносного движения. Обозначим толщину слоя перемещаемого материала через $h[5,6,7]$.

В качестве граничного условия движения материала примем, что при $z=0 v_{x}=v_{0}$, где $v_{0}$ - линейная скорость на максимальном радиусе вращающегося диска, м/с.

Запишем граничные условия для перемещаемого слоя материала: при $z=h ; v_{\mathrm{x}}=0$. Для начальных условий (при $t=0$ ) примем $U_{\mathrm{x}}=0$ $(0<z<h)$.

Рассматриваемое выражение с определёнными начальными и граничными условиями является уравнением параболического типа. Тогда уравнение (4) с учётом граничных и начальных условий является математической моделью неустановившегося процесса дозирования.

Далее заменим искомую функцию суммой двух функций:

$$
\begin{aligned}
& v(\mathrm{z}, \tau)=w(x)+u(\mathrm{z}, \tau), \quad \text { (5) } \\
& \text { где } w^{\prime \prime}(\mathrm{z})=0 \text {; функция } \\
& w(\mathrm{z}) \text { удовлет- }
\end{aligned}
$$
воряет граничным условиям:

$$
w(0)=v_{0} ; \quad w(h)=0
$$

а функция $u(x, \tau)$ удовлетворяет уравнению

$$
\frac{\partial u}{\partial \tau}=v \frac{\partial^{2} u}{\partial z^{2}}
$$

при следующих однородных граничных условиях:

$$
u(0, \tau)=0 ; \quad u(\mathrm{~h}, \tau)=0
$$

и при начальном условии

$$
\begin{aligned}
& v_{x}(\mathrm{z}, 0)=u(\mathrm{z}, 0)+w(\mathrm{z})=0 \\
& u(\mathrm{z}, 0)=-w(\mathrm{z}) .
\end{aligned}
$$

Окончательно решение уравнения (4) примет вид:

$v_{x}(z, t)=v_{0}\left(1-\frac{z}{h}-\frac{2}{\pi} \sum_{k=1}^{\infty} \frac{1}{k} \cdot e^{-\frac{v k^{2} \pi^{2}}{h^{2}} t} \sin \frac{k \pi}{h} z\right)$

С учётом того, что можно ограничиться двумя членами данного ряда, получим: 


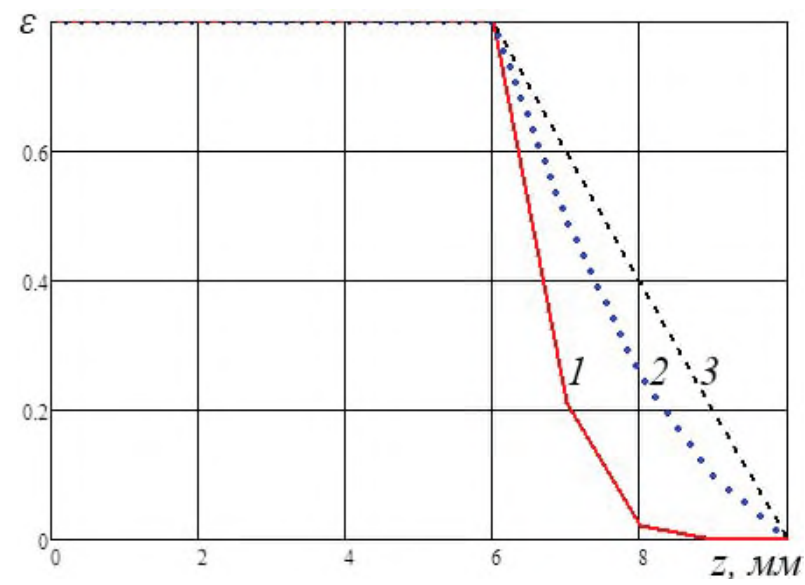

Рис. 4 - Зависимость отношения скоростей $\varepsilon$ от координаты $z$ в различные моменты времени: $1-t=1 \mathrm{c} ; 2-t=3 \mathrm{c} ; 3-t=10 \mathrm{c}$.

$v_{x}(z, t)=v_{0}\left[1-\frac{z}{h}-\frac{2}{\pi} \sin \frac{\pi}{h} z \cdot e^{-\frac{v \pi^{2}}{h^{2}} t}\left(1+e^{-\frac{3 v \pi^{2}}{h^{2}} t} \cdot \cos \frac{\pi}{h} z\right)\right]$.

Для анализа зависимости (11) определим отношение скоростей перемещения сыпучего материала к линейной скорости диска $\varepsilon(z, t)=v_{x}(z, t) / v_{0}$ по толщине слоя семян в различные моменты времени:

$\varepsilon=\frac{v_{x}(z, t)}{v_{0}}=\left[1-\frac{z}{h}-\frac{2}{\pi} \sin \frac{\pi}{h} z \cdot e^{-\frac{v \pi^{2}}{h^{2}} t}\left(1+e^{-\frac{3 v \pi^{2}}{h^{2} t}} \cdot \cos \frac{\pi}{h} z\right)\right]$

Из решения выражения (12) и условия, что $t$ находится пределах $0 . . .10$ с, следует, что режим движения материала является установившимся, а скорость по оси z

$$
v_{x}(z, t)=v_{0}\left(1-\frac{z}{h}\right) .
$$

Полученные выражения объясняют тот факт, что в дозирующее окно под козырёк вначале попадают частицы, располагающиеся рядом с окном, а затем - следующие за ними [8, 9, 10].

\section{Результаты исследований}

Результаты расчетов для различных случаев расположения штифтов дозирующего устройства приведены на рисунках 2...7. На рисунке 2 представлена зависимость отношения скоростей $\varepsilon=v_{x} / v_{0}$ от координаты $z$ вдоль оси по толщине перемещаемого слоя в различные моменты времени.

На рис. 3 показана зависимость изменения отношения скоростей $\varepsilon(z, t)=v_{x}(z, t) / v_{0}$ от координаты $z$ вдоль оси по толщине перемещаемого слоя и времени $t$.

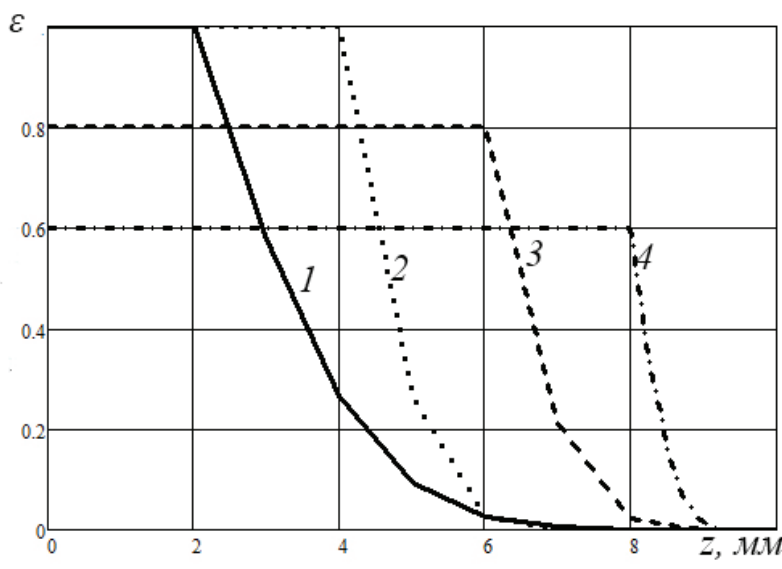

Рис. 5 - Зависимость отношения скоростей $\varepsilon$ от координаты $z$ при $\boldsymbol{t}=1$ с и различных высотах выхода штифтов $h$ : $1-h=2$ мм; $2-h=4 \mathrm{Mm} ; 3-h=6 \mathrm{Mm} ; 4-h=8 \mathrm{Mm}$

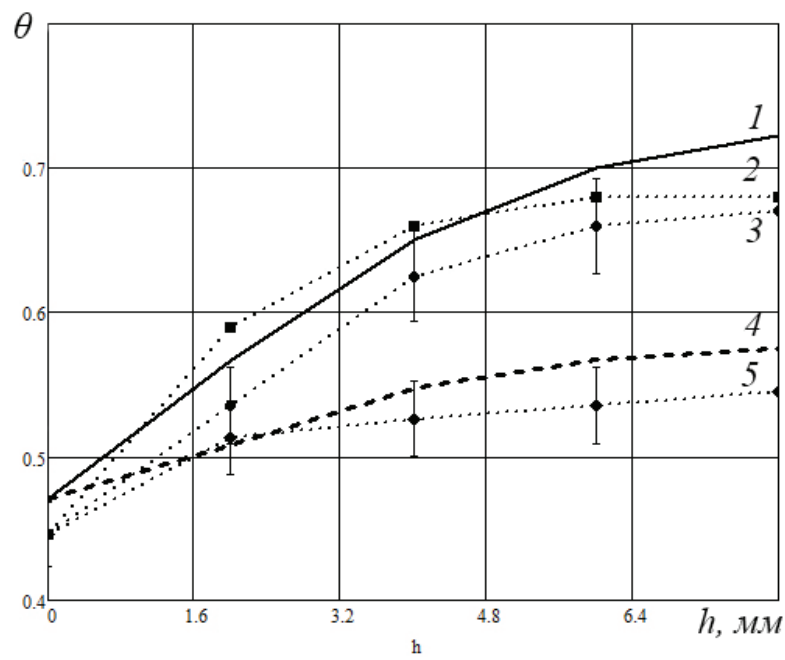

Рис. 6 - Изменение пропускной способности $\boldsymbol{\theta}$ от высоты выхода штифтов $\boldsymbol{h}$ : где 1,4 - теоретические зависимости; 2, 3, 5 - экспериментальные зависимости с различными вариантами расположения штифтов: 2 - в начале окна; 3 - в середине окна; 5 - в конце окна

\section{Зависимость отношения скоростей} $\varepsilon=v_{x} / v_{0}$ от координаты $z$ в различные моменты времени при выдвижении штифтов на высоту 6 мм показано на рис. 4.

Зависимость отношения скоростей $\varepsilon=v_{x} / v_{0}$ от координаты $z$ при $t=1$ с и выхода штифтов $h$ на высоту от 2 мм до 8 мм представлено на рис. 5. 
На рис. 6 показано изменение пропускной способности дозирующего устройства $\theta=Q / Q_{0}$ от высоты выхода штифтов $h$.

В результате анализа представленных зависимостей выявлено, что большая пропускная способность дозирующего устройства обеспечивается при высоте штифтов более 6 мм и их расположении в начале и в конце зоны подачи материала.

\section{Выводы}

Использование штифтов для истечения сыпучего материала из загрузочного окна в дисково-штифтовом дозирующем устройстве позволяет обеспечить значительное увеличение его пропускной способности. Проведенные теоретические исследования по определению скорости перемещения сыпучего материала вращающимся диском с подпружиненными штифтами позволяют получить оптимальную пропускную способность устройства при дозировании частиц материала в зависимости от конструктивных параметров этого устройства и физико-механических свойств материала. При максимальной высоте штифтов в средней части зоны загрузки материала пропускная способность устройства не превышает 0,55 кг/с.

\section{Библиографический список}

1. Крючин, Александр Николаевич. Повышение качества посева семян трав самоходной пневматической мини-сеялкой применением дисково-штифтового высевающего аппарата: автореф. дис. ... канд. технических наук: 05.20.01 / А.Н. Крючин. - Пенза, 2016. - 18 с.

2. Груздев, И.Э. Теории шнековых устройств / И.Э. Груздев, Р.Г. Мирзоев, В.И. Яиков. - Л.: Издво Ленинградского университета, 1978. - 144 с.
3. Гутьяр, Е.Я. Элементарная теория вертикального винтового транспортёра / Е.Я. Гутьяр // Научные труды МИМЭСХ им. В.М. Молотова. М.: Машгиз, 1956. - Том 2. - С. 8-12.

4. Желтов, В.П. Расчёт спиральных винтовых конвейеров / В.П. Желтов // Вестник машиностроения. - 1975. - № 5. - С. 18-21.

5. SolidWorks 2007/2008. Компьютерное моделирование в инженерной практике / А.А. Алямовский, А.А. Собачкин, Е.В. Одинцов, А.И. Харитонович, Н.Б. Пономарев. - Спб.: БХВПетербург, 2008. - 1040 с.

6. Математическое моделирование при оптимизации параметров сушильной установки контактного типа / В.И. Курдюмов, А.А. Павлушин, С.Г. Мударисов, В.И. Долгов // Пермский аграрный вестник. - 2016. - № 2(14). - С. 107- 112.

7. Начальные скорости движения частицы материала при перемещении спиральным винтом / Ю.М. Исаев, Х.Х. Губейдуллин, Н.М. Семашкин, И.И. Шигапов // Аграрная наука. - 2014. - № 10. - С. 28 - 30.

8. Semashkin, N.M. Revision of the cosmic distance duality tests / N.M. Semashkin, A.V. Nikolaev // Проблемы теоретической и наблюдательной космологии. 5-ая Ульяновская международная школа-семинар. - Ульяновск: ГПУ им. И.Н. Ульянова, 2016. - 69 с.

9. Optimization of research and methodology work at university in terms of the process approach / I.D. Ibragimov, R.R. Iskhakova, M.A. Galeeva, M.M. Kalashnikova, Yu.V. Ryseva, I.I. Galimzyanova, I.A. Sharonov // Journal of Sustainable Development. 2015. - Том 8, № 3. - С. 234-241.

10. Васильев, В.Ф. Водометные движители / В.Ф. Васильев. - М.:МАДИ ГТУ, 2006. - 45 с.

\title{
THEORETICAL RESEARCH OF THE PROCESS OF BULK MATERIAL DOSING
}

\author{
Semashkin N.M. ${ }^{1}$, Isaev Yu.M. ${ }^{1}$, Kryuchin N.P. ${ }^{2}$, Kryuchin A.N. ${ }^{2}$ \\ ${ }^{1}$ FSBEI HE Ulyanovsk State Agrarian University \\ ${ }^{2}$ FSBEI HE Samara State Agricultural Academy \\ 1432017, Ulyanovsk, Novyi Venets Boulevard, 1; tel .: 8 (84231) 55-95-49, e-mail: emotion.snm@mail.ru \\ 2446442, Samara region, Ust-Kinelskyv., Uchebnaya st., 2, e-mail: kryuchin@inbox.ru
}

Key words: particle, bulk material, Navier-Stokes equation, dosing, movement, throughput.

The article describes the movement of bulk material based on the hydrodynamic model using the Navier-Stokes equation for viscous fluid in vector form. A layer of transported material is studied, its thickness is significantly smaller compared to its length and width. The theoretical part of the article describes studies of the movement of bulk material in a dosing device, which takes into account the area and mass of the material. The average speed of the particles of the material is limited by the height of the raising of pins, which differs from the speed of disk rotation, since one of the main characteristics of the bulk material is the coefficient of internal friction of the particles of the material. At the same time, to determine the throughput of the measuring hopper, an equasion was taken into account to determine the average speed of movement of particles of a material. An equation was derived that represents a mathematical model of an unsteady motion regime and it was analyzed taking into account boundary and initial conditions. The ratio of the velocity of the bulk material to the linear velocity of the conveyor at various points along the thickness of the layer of the material being moved at different time is found. As a result, it was revealed that the use of pins for the flow of bulk material from the loading chamber of the disk-pin dosing device allows to provide a significant increase in its throughput. The obtained theoretical studies of the movement speed specification of the bulk material by a rotating disk with spring pins allow to obtain a suitable throughput of the device when dosing particles of the material depending on the design parameters of this device and the physical and mechanical properties of the material. With the maximum height of the pins in the middle part of the material loading zone, the throughput of the device did not exceed $0.55 \mathrm{~kg} / \mathrm{s}$. 
Bibliography

1. Kryuchin, Alexander Nikolaevich. Improving the sowing quality of grass seeds with self-propelled pneumatic mini-seeder using disc-pin seeding device: author's abstract of dissertation of Candidate of Technical Sciences: 05.20.01 / A.N. Kryuchin. - Penza, 2016. - 18 p.

2. Gruzdev, I.E. Theories of screw devices / I.E. Gruzdev, R.G. Mirzoev, V.I. Yaikov. - L.: Publishing House of the University of Leningrad, 1978. - 144 p.

3. Gutyar, E.Ya. Elementary theory of vertical screw conveyor / E.Ya. Gutyar //Scientific works of Moscow Institute of Mechanization and Electrification of Agriculture named after V.M. Molotov. - M .: Mashgiz, 1956. - Volume 2. - P. 8-12.

4. 10. Zheltov, V.P. Calculation of spiral screw conveyors / V.P. Zheltov //Vestnik of mechanical engineering. - 1975. - No 5. - P. 18-21.

5. SolidWorks 2007/2008. Computer modeling in engineering practice/A.A. Alyamovsky, A.A. Sobachkin, E.V. Odintsov, A.I. Kharitonovich, N.B. Ponomarev. - SPb .: BKhV-Petersburg, 2008. - 1040 p.

6. Mathematical modeling when improving the parameters of a contact-type drying installation / V.I. Kurdyumov, A.A. Pavlushin, S.G. Mudarisov, V.I. Dolgov // Perm Agrarian Journal. - 2016. - № 2 (14). - P. 107- 112.

7. The initial velocity of the particle of the material when moved by a spiral screw / Yu.M. Isaev, Kh.Kh. Gubeidullin, N.M. Semashkin, I.I. Shigapov // Agrarian science. - 2014. - № 10. - P. 28 - 30.

8. Semashkin, N.M. Revision of the cosmic distance duality tests / N.M. Semashkin, A.V. Nikolaev // Problems of theoretical and observational cosmology. 5th Ulyanovsk International training Seminar. - Ulyanovsk: Teacher Training University named after I.N. Ulyanov, 2016. - 69 p.

9. Optimization of research and methodology work at university in terms of the process approach / I.D. Ibragimov, R.R. Iskhakova, M.A. Galeeva, M.M. Kalashnikova, Yu.V. Ryseva, I.I. Galimzyanova, I.A. Sharonov // Journal of Sustainable Development. - 2015. - Volume 8, No. 3. - P. $234-241$.

10. Vasiliev, V.F. Water jets /V.F. Vasiliev. - M.: Moscow Automobile and Highway State Technical University, 2006. - 45 p. 